\title{
The Influence of Traumatic Axonal Injury in Thalamus and Brainstem on Level of Consciousness at Scene or Admission: A Clinical Magnetic Resonance Imaging Study
}

\author{
Hans Kristian Moe, Kent Gøran Moen,, Toril Skandsen,,3 Kjell Arne Kvistad, \\ Steven Laureys, Asta Håberg, ${ }^{1,4}$ and Anne Vik ${ }^{1,6}$
}

\begin{abstract}
The aim of this study was to investigate how traumatic axonal injury (TAI) lesions in the thalamus, basal ganglia, and brainstem on clinical brain magnetic resonance imaging (MRI) are associated with level of consciousness in the acute phase in patients with moderate to severe traumatic brain injury (TBI). There were 158 patients with moderate to severe TBI (7-70 years) with early 1.5T MRI (median 7 days, range $0-35$ ) without mass lesion included prospectively. Glasgow Coma Scale (GCS) scores were registered before intubation or at admission. The TAI lesions were identified in $\mathrm{T} 2 *$ gradient echo, fluid attenuated inversion recovery, and diffusion weighted imaging scans. In addition to registering TAI lesions in hemispheric white matter and the corpus callosum, TAI lesions in the thalamus, basal ganglia, and brainstem were classified as uni- or bilateral. Twenty percent of patients had TAI lesions in the thalamus (7\% bilateral), $18 \%$ in basal ganglia (2\% bilateral), and $29 \%$ in the brainstem ( $9 \%$ bilateral). One of 26 bilateral lesions in the thalamus or brainstem was found on computed tomography. The GCS scores were lower in patients with bilateral lesions in the thalamus (median four) and brainstem (median five) than in those with corresponding unilateral lesions (median six and eight, $p=0.002$ and 0.022). The TAI locations most associated with low GCS scores in univariable ordinal regression analyses were bilateral TAI lesions in the thalamus (odds ratio [OR] 35.8; confidence interval [CI: 10.5-121.8], $p<0.001$ ), followed by bilateral lesions in basal ganglia (OR 13.1 [CI: 2.0-88.2], $p=0.008)$ and bilateral lesions in the brainstem (OR 11.4 [CI: 4.0-32.2], $p<0.001$ ). This Trondheim TBI study showed that patients with bilateral TAI lesions in the thalamus, basal ganglia, or brainstem had particularly low consciousness at admission. We suggest these bilateral lesions should be evaluated further as possible biomarkers in a new TAI-MRI classification as a worst grade, because they could explain low consciousness in patients without mass lesions.
\end{abstract}

Keywords: basal ganglia; brainstem; craniocerebral trauma; Glasgow Coma Scale; neuroimaging; thalamus

\section{Introduction}

$\mathbf{T}$ RAUMATIC AXONAL INJURY (TAI) is one of the most important types of pathology in traumatic brain injury (TBI) ${ }^{1,2}$ and occurs when the head is exposed to acceleration-deceleration and rotational forces. The terminology has changed from shearing injury to diffuse axonal injury or diffuse TBI and is now increasingly called TAI also in patients. Clinical magnetic resonance imaging (MRI) shows indirect signs of TAI and has superior detection sensitivity compared with computed tomography (CT), where such lesions remain largely undetectable. ${ }^{3}$ TAI can be divided into three grades based on location of lesions in cerebral white matter on clinical MRI ${ }^{4}$ : TAI in the hemispheres/cerebellum (grade 1), TAI in the corpus callosum (grade 2), and TAI in the brainstem (grade 3). This standard grading is assumed to reflect the severity of TAI. ${ }^{5}$

The classification of TAI is based on white matter lesions, but similar lesions can also be found in subcortical nuclei, such as the thalamus and the basal ganglia. ${ }^{6,7}$ A recent study from our research

\footnotetext{
${ }^{1}$ Department of Neuromedicine and Movement Science, Norwegian University of Science and Technology (NTNU), Trondheim, Norway.

${ }^{2}$ Department of Medical Imaging, Levanger Hospital, Levanger, Norway.

Departments of ${ }^{3}$ Physical Medicine and Rehabilitation and ${ }^{4}$ Radiology and Nuclear Medicine, St. Olavs University Hospital, Trondheim, Norway.

${ }^{5}$ Coma Science Group, Cyclotron Research Center and University Hospital of Liège, University of Liège, Liège, Belgium.

${ }^{6}$ Department of Neurosurgery, St. Olavs University Hospital, Trondheim, Norway.
}

(c) Hans Kristian Moe et al., 2018; Published by Mary Ann Liebert, Inc. This Open Access article is distributed under the terms of the Creative Commons Attribution Noncommercial License (http://creativecommons.org/licenses/by-nc/4.0/) which permits any noncommercial use, distribution, and reproduction in any medium, provided the original author(s) and the source are credited. 
group has shown that MRI lesion load in the thalamus was an important independent predictor of outcome, and that such lesions were consistently associated with TAI in the white matter. ${ }^{8}$ Hence, we suggest labeling these gray matter lesions TAI lesions.

An important step in the diagnosis, management, and prognostication of TBI in patients is to assess their state of consciousness, which is performed with the Glasgow Coma Scale (GCS) score. ${ }^{9}$ Consciousness is not fully understood, but arousal (i.e., eye opening) is considered to depend on functions in the brainstem and thalamus, while awareness (e.g., response to command and orientation to pain) is considered to depend on functional connections between the aforementioned structures, plus the basal ganglia and the cortex. ${ }^{10-12}$ The involved anatomical structures are located in both hemispheres and to the left and right side of the brainstem.

In patients with TBI, there are several possible reasons for a reduced level of consciousness and low GCS score at the scene or admission. First, increased intracranial pressure (ICP)—e.g., because of mass lesions - may reduce the GCS score. Second, TAI can reduce the GCS score as shown in moderate to severe TBI comparing patients with and without TAI lesions. ${ }^{1}$ Also, brainstem lesions (mostly TAI lesions) have been detected in almost half of the patients with severe TBI surviving the acute phase, ${ }^{13}$ suggesting importance for reduced consciousness. Finally, micro-hemorrhagic and nonhemorrhagic MRI lesions in the subcortical nuclei have been shown to be associated with decreased GCS scores in two small studies. ${ }^{14,15}$ In addition, systemic factors such as intoxication, hypotension, and hypoxia may reduce the GCS score.

Several previous studies have investigated the relationship between the GCS score in the acute phase and different kinds of MRI findings in the more chronic phase. ${ }^{16-20}$ It has been shown recently, however, that MRI findings will attenuate during the first months. ${ }^{21}$ Hence, it is important to explore whether some specific MRI lesions in the early phase can explain reduced GCS scores in patients with modest CT findings. Such lesions could be MRI biomarkers of importance for clinicians in the acute phase and also be part of future, more comprehensive prognostic models. To our knowledge, no previous study has investigated how unilateral versus bilateral TAI lesions in the brainstem (TAI grade 3) and subcortical nuclei detected on MRI can influence consciousness in patients with TBI in the very acute phase.

Thus, the aim of this clinical MRI study is to investigate whether uni- and bilateral TAI lesions in the thalamus, basal ganglia, and brainstem are associated with the GCS score at the scene or admission in patients with moderate and severe TBI without major mass lesions.

\section{Methods}

\section{Patients}

Inclusion criteria were: Patients aged 7-70 years admitted to St. Olavs Hospital, Trondheim University Hospital, Norway, who according to the Head Injury Severity $\mathrm{Scale}^{22}$ were classified as moderate (GCS 9-13, or GCS 14-15 and loss of consciousness $>5$ min [two patients]) and severe TBI (GCS $\leq 8$, Fig. 1). Only those who underwent early $1.5 \mathrm{~T}$ MRI assessment using a standardized clinical research MRI protocol ${ }^{1}$ were included. From October 2004 to October 2013 a total of 396 patients (7-70 years old) with moderate and severe TBI were admitted, of which 222 patients underwent MRI within five weeks post-injury and were considered for inclusion in the present study.

Exclusion criteria were: (1) age younger than 7 years, to ensure reliable GCS scoring; (2) age older than 70 years because of the

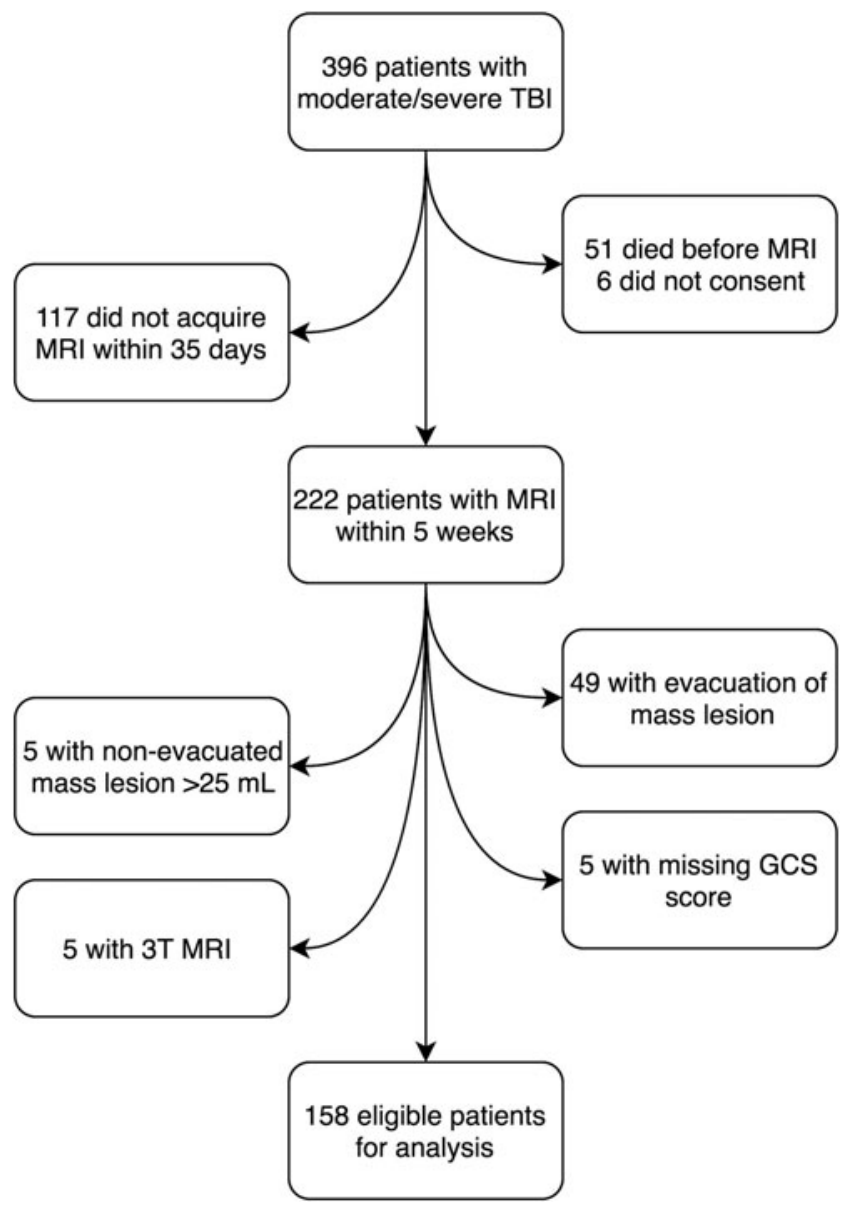

FIG. 1. Flowchart of the 158 included patients. TBI, traumatic brain injury; MRI, magnetic resonance imaging; GCS, Glasgow Coma Scale.

higher prevalence of nonspecific white matter lesions in the elderly population $^{23}$; (3) evacuation of mass lesion ( $n=49$, Marshall CT score 5) or nonevacuated mass lesions $>25 \mathrm{~mL}(n=5$, Marshall CT score 6) - to reduce the influence of increased ICP on GCS scores; (4) 3T MRI examination $(n=5)$; or $(5)$ missing GCS score $(n=5)$. This left 158 patients eligible for analyses. All patients were included prospectively in the Trondheim TBI Studies, and the methodology for the collection of demographic and injury-related variables is described in previous publications. ${ }^{1,21}$

\section{GCS score}

For intubated patients, the pre-intubation GCS score was used, while for the other patients, the admission GCS score at Trondheim University Hospital was used. All GCS scores were collected in nonsedated patients. The GCS scores were collected at the scene of injury by anesthesiologists $(n=73)$, at the primary hospital by consultants/residents in general surgery $(n=18)$, or on admission at Trondheim University Hospital by consultants/residents in neurosurgery or neurology $(n=67)$. The GCS score at either the primary hospital or at the university hospital was assessed at median $60 \mathrm{~min}$ post-injury (interquartile range [IQR] $30-90,7 \%$ after $5 \mathrm{~h}$ [unknown time of injury $n=10$, missing $n=4]$ ). If the GCS score of the patient rose significantly during the first few hours and stabilized, as a sign of alcohol intoxication, the highest GCS score was used $(n=5)$. The eye component of the GCS score was also registered (missing $n=8$ ). 


\section{Other injury variables, blood alcohol concentration, and outcome}

Injury Severity Score (ISS) was estimated, and pupillary dilatation, secondary events such as pre-hospital or admission hypoxia and hypotension were registered. This was performed at any time point before or at admission-hence, not necessarily at the same time as the GCS score assessment. Because blood alcohol concentration (BAC) may influence the GCS score, ${ }^{24}$ BAC was analyzed and a cutoff value of $>100 \mathrm{mg} / \mathrm{dL}$ for intoxication was chosen in the comparison between included and nonincluded patients. ${ }^{25} \mathrm{~A}$ radiologist or resident in neurosurgery/radiology in collaboration with three neuroradiologists reviewed all the admission CT scans (first CT). Compressed or absent basal cisterns were described to evaluate signs of increased ICP and the Marshall- and Rotterdam CT scores were used. ${ }^{26,27}$

A.M.F (radiologist) reviewed the first and worst CT scans for any uni- or bilateral lesions in the thalamus, brainstem, and basal ganglia for all patients with such TAI lesions on MRI. In addition, 10 patients without TAI lesions in these subcortical structures on MRI were both randomly chosen and added to the mix of images. Worst CT was defined as the CT scan (first CT or follow-up CT) that yielded the worst Marshall CT score. Marshall 5 and 6 were regarded equally as the worst score.

The radiologist was blinded to the MRI findings and their distribution among the patients as well as to all other information except for age and date of CT. Head injury-related global outcome was assessed 12 months post-injury by the Glasgow Outcome Scale Extended (GOSE). ${ }^{8,28}$

\section{$M R I$}

The MRI examinations were performed either at St. Olavs Hospital or at one of its referral hospitals. The 1.5 Tesla scanners (Siemens symphony or Siemens Avanto; Siemens Medical, Erlangen Germany) with a six-channel head coil were used. The previously described ${ }^{1}$ clinical TBI MRI protocol consisted of sagittal turbo spin echo (TSE) T2 imaging, sagittal, transverse T2* weighted gradient echo imaging (GRE), coronal and transverse T2 fluid attenuated inversion recovery (FLAIR) imaging, and diffusion weighted imaging (DWI).

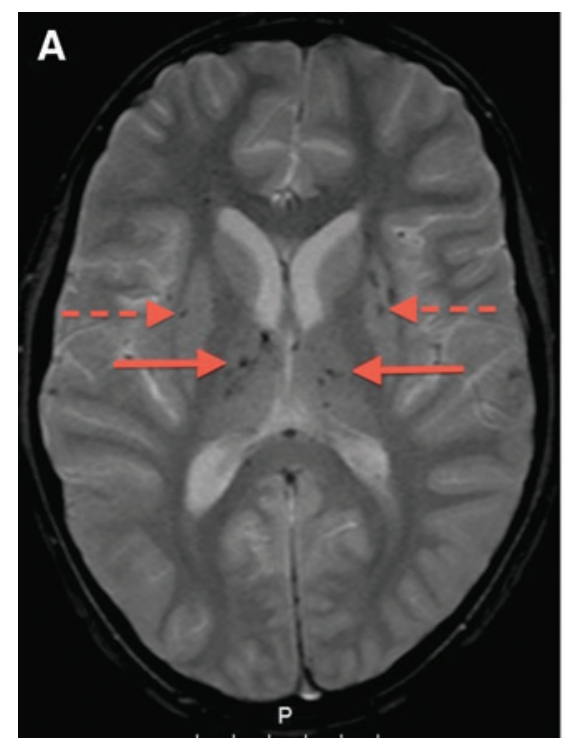

\section{Image reading}

For 138 of the patients, K.G.M. (resident in radiology), K.A.K, J.R., and M.F, (all experienced consultant neuroradiologists) read the MRIs in accordance with pre-defined variables. ${ }^{21}$ All were blinded to patient identification, clinical information, and time of examination. For the last 20 patients J.X. (resident in radiology) characterized the image findings in collaboration with K.G.M, K.A.K., and M.F., using the same procedures. Interrater analysis for classification of TAI grade was performed in an earlier study (weighted Cohen $\kappa 0,74,95 \%$ CI $0.69-0.80){ }^{21}$

The TAI lesions were described according to their location-as lesions in hemispheric white matter, corpus callosum, thalamus, basal ganglia, and brainstem (Fig. 2). Lesions in the thalamus, basal ganglia and brainstem were classified as uni- or bilateral. The presence of lesions in the different imaging sequences-i.e., T2*GRE, FLAIR, and DWI-were recorded. Periventricular signal hyperintensities ("caps and bands") that were considered part of the normal aging were not registered as $\mathrm{TAI},{ }^{29}$ nor were other nonspecific white matter hyperintensities, ${ }^{23,30}$ or traumatic lesions acquired during a surgical procedure (e.g., from insertion of intracranial pressure devices). Superficial lesions in the cortex were defined as contusions.

\section{Statistical analyses}

Patient- and injury characteristics are presented as percentages, mean and median with IQR (25th to 75 th percentile). Mann Whitney $U$ test was used for comparisons of medians between groups (GCS score and other variables without normal distribution). Spearman rho was used to assess correlation between the eye component of the GCS score and the total GCS score, and Fisher exact test was used for comparison of proportions. Cohen $\kappa$ (ranging from 0 to 1 , where 1 is perfect agreement) was used to assess the agreement between MR and CT findings in the thalamus, basal ganglia, and brainstem. Ordinal univariable regression analyses with inverted GCS score as the dependent variable were performed to assess its association with TAI lesions in different locations, where no visible lesion in the location in question was coded as the reference category. Rotterdam CT score was analyzed as a scalar variable.

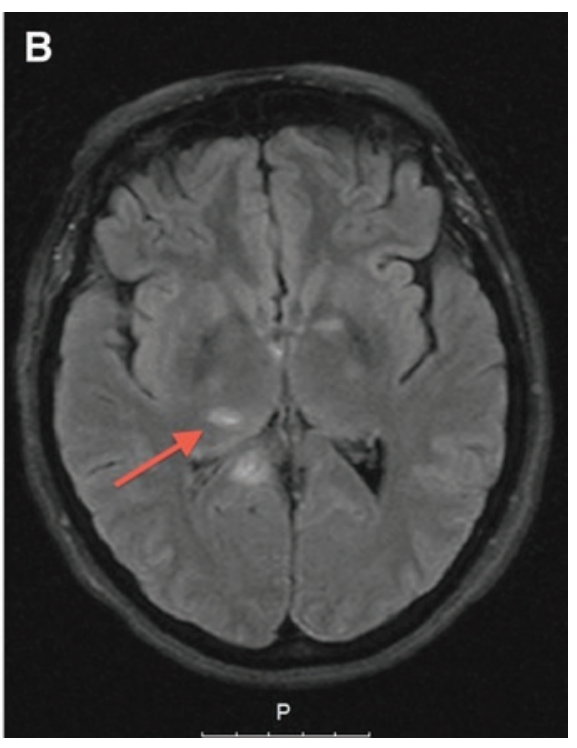

FIG. 2. Magnetic resonance imaging of patients with traumatic axonal injury (TAI) lesions: (A) T2* gradient echo image showing multiple bilateral lesions in the thalamus (unbroken arrows) and basal ganglia (broken arrows). (B) Fluid attenuated inversion recovery image showing multiple TAI lesions (arrow shows TAI lesion in the right thalamus). 
We chose not to perform multivariable analyses because we consider this to be an explorative study. Adjustment for other injury variables in the acute phase may also be challenging when studying association to GCS score. Also, because TAI lesions in different locations are highly correlated, lesion locations were analyzed separately to avoid multicollinearity. All analyses were performed with the IBM Statistical Package for the Social Sciences (SPSS) Statistics V.21 or STATA/IC V.13.1. Threshold for significance was $p<0.05$, two-tailed.

\section{Ethics}

The Regional Committee for Medical Research Ethics approved the study, and written consent was obtained from the patients or, when incapacitated, from their next of kin.

\section{Results}

\section{Demographic- and injury-related variables} in the consecutive TBI cohort

Characteristics of the 158 patients included in this MRI study are shown in Table 1. Of the 238 nonincluded patients, $45 \%$ had Marshall score 5-6 (intracranial mass lesion). There were also several other differences between the included and nonincluded patients.

\section{Occurrence of TAl lesions in different locations of the brain}

Clinical MRI was performed at median seven days (range 0-35), which was within two weeks for $77 \%$ of the 158 patients. Visible TAI lesions were depicted in $128(82 \%)$ patients. There were $20 \%$ who had TAI lesions in the thalamus, $18 \%$ who had TAI lesions in the basal ganglia, and 29\% who had TAI lesions in the brainstem (TAI grade 3, Table 2). Bilateral lesions were detected in the thalamus in $11(7 \%)$ patients, in the basal ganglia in three $(2 \%)$ patients, and in the brainstem in $15(9 \%)$ patients. All of the patients with TAI lesions in the thalamus or basal ganglia had TAI lesions elsewhere in the brain. Patients who had MRI 0-7 days post-injury $(n=85)$ had higher GCS scores than those who had MRI later $(n=73, p=0.002)$, but there was no difference in age, TAI grade, or the occurrence of bilateral lesions in the thalamus, basal ganglia, or brainstem ( $p$ 0.348-1).

Only one of the $11(9 \%)$ patients with bilateral TAI in the thalamus on MRI had bilateral thalamic findings on the CT scan (Table 3). None of the $15(0 \%)$ patients with bilateral TAI in the brainstem on MRI had bilateral brainstem findings on the CT scan. First and worst CT scans yielded the same results in all patients, except in one patient who had a unilateral lesion in the thalamus only on first CT and one patient with a unilateral brainstem lesion only on worst CT.

\section{GCS score in relation to presence of uni- and bilateral TAl lesions}

We found lower GCS scores in patients with bilateral lesions in the thalamus (median 4, IQR 3-4) and brainstem (median 5, IQR $3-$ 7) than in those with unilateral lesions in these locations (median 6 , IQR 5-10, $p=0.002$ and median 8, IQR 5-10, $p=0.022$, respectively, Fig. 3A, 3C). Patients with bilateral TAI in the thalamus also had worse outcome-i.e., lower GOSE scores (median 3, IQR

Table 1. Patient Characteristics, Injury-Related Variables, and Outcome in the Included and The Nonincluded Patients in the Consecutive Traumatic Brain Injury Cohort

\begin{tabular}{|c|c|c|c|}
\hline Variable & $\begin{array}{l}\text { Included patients } \\
\quad(\mathrm{n}=158)\end{array}$ & $\begin{array}{l}\text { Nonincluded patients } \\
\qquad(\mathrm{n}=238)\end{array}$ & $\mathrm{p}$ value \\
\hline Age (median, IQR) & $26.7(19.2-44.9)$ & $41.6(23.8-56.0)$ & $<0.001$ \\
\hline Sex (male/female, \%) & $118 / 40(75 / 25)$ & $187 / 51(79 / 21)$ & 0.394 \\
\hline \multicolumn{4}{|l|}{ Injury mechanism (\%) } \\
\hline Vehicle accident & $96(61)$ & $88(37)$ & $<0.001$ \\
\hline Fall & $42(27)$ & $115(48)$ & $<0.001$ \\
\hline Other & $19(12)$ & $29(12)$ & 1.0 \\
\hline Unknown & $1(0.6)$ & $6(3)$ & 0.251 \\
\hline Severe TBI $(\%)$ & $68(43)$ & $141(59)$ & 0.001 \\
\hline GCS score (median, IQR) & $9(6-12)$ & $7(4-13)$ & 0.040 \\
\hline Hypoxia $\left(\mathrm{O}_{2} \text { sat }<92\right)^{\mathrm{a}}$ & $28(18)$ & $52(22)$ & 0.371 \\
\hline Hypotension (systolic blood pressure $<90 \mathrm{~mm} \mathrm{Hg})^{\mathrm{a}}$ & $19(12)$ & 45 (19) & 0.071 \\
\hline \multicolumn{4}{|l|}{ Pupil dilatation at admission (\%) } \\
\hline Unilateral dilatation & $12(8)$ & $36(15)$ & $\mathbf{0 . 0 2 8}$ \\
\hline Bilateral dilatation & $2(1)$ & $29(12)$ & $<0.001$ \\
\hline ISS (median, IQR) & $25(17-33)$ & $25(17-33)$ & 0.842 \\
\hline $\mathrm{BAC} \geq 100 \mathrm{mg} / \mathrm{dL}(\%)^{\mathrm{b}}$ & $38(30)$ & $77(41)$ & 0.044 \\
\hline \multicolumn{4}{|l|}{ Marshall CT score $(\%)$} \\
\hline $1-2$ & $132(84)$ & $95(40)$ & $<0.001$ \\
\hline $3-4$ & $25(16)$ & $43(18)$ & 0.498 \\
\hline $5-6$ & $0(0)$ & $89(37)$ & $<0.001$ \\
\hline Rotterdam CT score $\left(\right.$ mean, IQR) ${ }^{\mathrm{c}}$ & $2.6(2-3)$ & $3.4(2-4)$ & $<0.001$ \\
\hline Mortality at 12 months (\%) & $2(1)$ & $58(24)$ & $<0.001$ \\
\hline GOSE score at 12 months (median, IQR) ${ }^{\mathrm{d}}$ & $7(5-8)$ & $6(3-8)$ & $<0.001$ \\
\hline
\end{tabular}

IQR, interquartile range; TBI, traumatic brain injury; GCS, Glasgow Coma Scale; ISS, Injury Severity Score; BAC, blood alcohol concentration; CT, computed tomography; GOSE, Glasgow Outcome Scale Extended.

${ }^{\mathrm{a}}$ Pre-hospitally or at admission.

${ }^{\mathrm{b}} 126$ of the included patients were tested for BAC $(81 \%)$.

${ }^{\mathrm{c}}$ Mean is presented, because median Rotterdam CT score was 3 for both groups.

${ }^{\mathrm{d}} 6$-month GOSE score was used if the 12-month GOSE score was missing $(n=8)$. Seven patients died of a cause other than the TBI, three patients were not possible to assess, and 23 patients were either not planned to or lost to follow-up in the total cohort of 396 patients. 
Table 2. Patients with Traumatic Axonal Injury Lesions Stratified into Location and Magnetic Resonance InJury SEQuence

\begin{tabular}{|c|c|c|c|c|}
\hline & $\begin{array}{l}\text { Any MRI } \\
\text { sequence } \\
(\mathrm{n}=158)\end{array}$ & $\begin{array}{c}T 2 * G R E \\
M R I \\
(\mathrm{n}=151)\end{array}$ & $\begin{array}{c}\text { FLAIR } \\
M R I \\
(\mathrm{n}=158)\end{array}$ & $\begin{array}{c}D W I \\
M R I \\
(\mathrm{n}=154)\end{array}$ \\
\hline TAI lesions & $\mathrm{n}(\%)$ & $\mathrm{n}(\%)$ & $\mathrm{n}(\%)$ & $\mathrm{n}(\%)$ \\
\hline $\begin{array}{l}\text { Hemispheric } \\
\text { white matter }\end{array}$ & $125(79)$ & $115(76)$ & $92(58)$ & $33(21)$ \\
\hline Corpus callosum & $84(53)$ & $64(42)$ & $71(45)$ & $46(30)$ \\
\hline Thalamus & $31(20)$ & $23(15)$ & $18(13)$ & $10(6)$ \\
\hline Basal ganglia & 28 (18) & $25(17)$ & $10(6)$ & $4(3)$ \\
\hline Brain stem & $46(29)$ & $31(21)$ & 30 (19) & $17(11)$ \\
\hline
\end{tabular}

TAI, traumatic axonal injury; MRI, magnetic resonance imaging; T2*GRE, T2 weighted gradient echo imaging; FLAIR, fluid attenuated inversion recovery imaging; DWI, diffusion weighted imaging. Seven and four patients lacked the T2*GRE and DWI sequences, respectively.

3-4.3) than patients with unilateral TAI in the thalamus (median 6, IQR 3-8, $p=0.009$ ).

Patients with unilateral lesions in the thalamus, basal ganglia (median 7, IQR 4.5-11), or brainstem also had lower GCS scores than patients without corresponding lesions (median 10 [IQR 712], median 10 [IQR 7-12] and median 11 [IQR 7-13], respectively; $p=0.005-0.011$, Fig. 3). Several of the patients with unilateral TAI lesions in deeper parts of the brain had GCS scores $>8$ : $40 \%$ of the 20 patients with unilateral TAI in the thalamus, $36 \%$ of the 25 patients with unilateral TAI in basal ganglia, and $48 \%$ of the 31 patients with unilateral TAI in the brainstem. Of the patients with GCS score $13(n=30), 30 \%$ had unilateral lesions in the thalamus, basal ganglia, and/or brainstem. There was no difference in the GCS score between patients with $(n=110)$ and those without contusions $(n=48)$ on MRI $(p=0.365)$.

The eye component of the GCS score had a very strong correlation with the total GCS score (Spearman rho $=0.877, p<0.001$ ). Of the 22 patients who had bilateral TAI lesions in the thalamus, basal ganglia, and/or brainstem, only three (14\%) patients had any form of eye opening (all three had bilateral brainstem lesions).

\section{Multiplicity of bilateral TAI lesions in deep brain structures and GCS scores}

Four patients had bilateral lesions in both the thalamus and brainstem, with a median GCS score of 3 (IQR 3-6, Fig. 4). Patients with bilateral TAI only in the thalamus had a median GCS score of
4 (IQR 3-4, $n=7$ ), while patients with bilateral TAI only in the brainstem had a median GCS score of 6 (IQR 3-10, $n=11$, $p=0.093)$.

\section{GCS score in relation to location of uni- and bilateral TAI lesions by MRI sequence}

Patients with bilateral thalamic lesions had lower median GCS scores than those with unilateral lesions for both T $2 *$ GRE (median 4 vs. $6.5, p=0.038$ ) and FLAIR (median 3 vs. $6, p=0.005$, Table 4). Patients with bilateral brainstem lesions detected by T2*GRE sequence had lower median GCS scores than patients with unilateral brainstem lesions (median 4.5 vs. 9, $p=0.008$ ), but no such difference was found for patients with bilateral brainstem lesions detected by FLAIR (median 5 vs. 7.5, $p=0.126$ ).

Patients with unilateral lesions in thalamus, basal ganglia, or brainstem had lower GCS scores than patients without such lesions in most imaging sequences (Table 4). Lesions in the thalamus, basal ganglia, or brainstem were detected more seldom by DWI than with T2*GRE (Fischer exact: $p=0.001-0.028$, Table 2 ).

\section{Univariable analyses of GCS score}

The highest OR for having a lower GCS score was found for bilateral lesions in the thalamus (OR 35.8), followed by bilateral lesions in the basal ganglia (OR 13.1) and brainstem (OR 11.4, Table 5). The BAC or compression of basal cisterns was not associated with lower GCS scores.

\section{Discussion}

This prospective clinical MRI study of moderate and severe patients with TBI without mass lesions demonstrated that patients with TAI lesions bilaterally in the thalamus, basal ganglia, or brainstem had particularly low consciousness at scene or admission. Indeed, none of these patients had eye opening, except three patients with bilateral TAI in the brainstem. Lesions in these locations were seldom detected on CT scans. Further, patients with unilateral lesions in the thalamus and brainstem had higher median GCS scores than those with the corresponding bilateral lesions. Finally, patients with unilateral lesions in the thalamus, basal ganglia, and/or brainstem had lower GCS scores than those without such lesions. The results of this study underscore the importance of detection of TAI lesions in subcortical nuclei and brainstem with early MRI for understanding low GCS scores in patients with modest intracranial findings on CT.

Table 3. Computed Tomography Findings in the 63 Patients with Traumatic Axonal Injury Lesions in the Thalamus, Basal Ganglia, and/or Brainstem on Magnetic Resonance Imaging

\begin{tabular}{|c|c|c|c|c|c|c|c|}
\hline \multirow[b]{2}{*}{ Lesion location } & \multicolumn{3}{|c|}{ MRI } & \multicolumn{3}{|c|}{$C T^{\mathrm{a}}$} & \multirow[b]{2}{*}{ Cohen $k$} \\
\hline & None & Unilateral & Bilateral & None & Unilateral & Bilateral & \\
\hline Thalamus & 32 & 20 & 11 & 57 & 5 & 1 & 0.10 \\
\hline Basal ganglia & 35 & 25 & 3 & 51 & 10 & $2^{\mathrm{b}}$ & 0.25 \\
\hline Brainstem & 17 & 31 & 15 & 60 & 3 & 0 & 0.01 \\
\hline
\end{tabular}

MRI, magnetic resonance imaging; CT, computed tomography.

${ }^{a}$ The 10 randomly added patients without MRI findings in subcortical structures also were all without such lesions on CT and are not presented in the table.

${ }^{b}$ Lesions in the basal ganglia on CT scan could not be identified as TAI lesions on MRI in two patients. One patient had uncertain findings on CT; the second also had a unilateral hemorrhage $>10 \mathrm{~mm}$. 

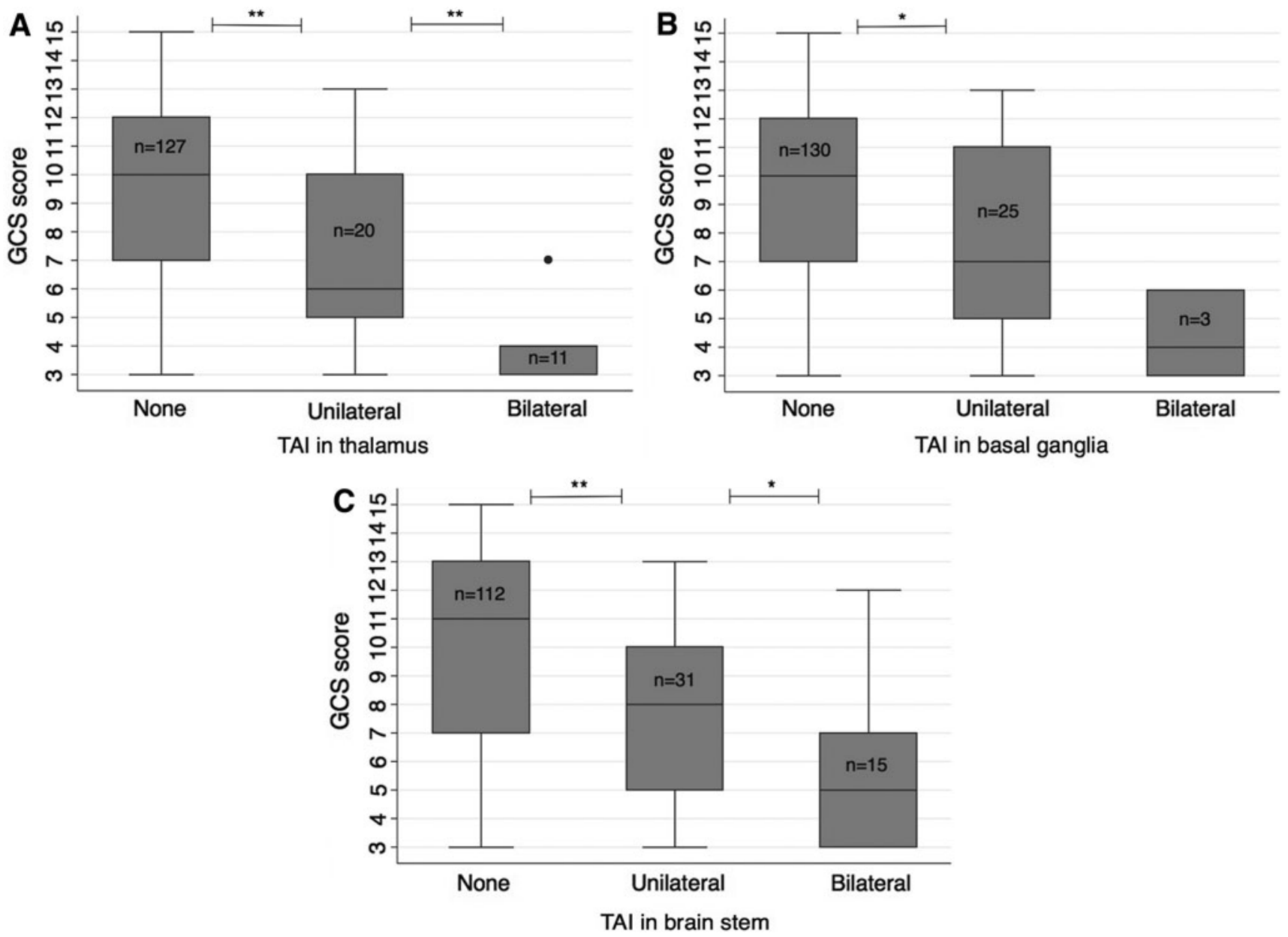

FIG. 3. The Glasgow coma Scale (GCS) score and the relation to uni-and bilateral traumatic axonal injury (TAI) lesions (any magnetic resonance imaging sequence) in the (A) thalamus, $(\mathbf{B})$ basal ganglia, and $(\mathbf{C})$ brainstem. ${ }^{*} p=0.01-0.05, * * p<0.01$.

\section{GCS score in relation to location of uni- and bilateral TAl lesions}

The finding that bilateral TAI lesions in the thalamus, basal ganglia, or brainstem were associated with particularly reduced GCS scores is in accordance with the importance of the ascending reticular activating system (ARAS) and the thalamus for consciousness. Consciousness can be divided into wakefulness and awareness of self and of external stimuli ${ }^{31}$-i.e., awareness cannot exist without wakefulness. Animal studies suggest that multiple brain structures are important for wakefulness, including cholinergic nuclei in the brainstem and basal forebrain, monoaminergic pathways in the brainstem and the posterior hypothalamus. ${ }^{32}$ Some of these pathways are modulated by the thalamus in close conjunction with the brainstem ARAS, ${ }^{33}$ while others pass directly from the ARAS and to the cerebral cortex or via the basal forebrain or basal ganglia. ${ }^{34,35}$

Our findings that patients with lesions in the thalamus and basal ganglia had decreased GCS scores are in accordance with the MRI study of Gerber and associates ${ }^{15}$ (T2 SE and T2*GRE within eight weeks post-injury) of 43 patients, demonstrating that lower GCS scores were associated with the number of both micro-hemorrhagic and other types of lesions in subcortical gray matter. In another clinical MRI study (T2 SE, T2*GRE, FLAIR and DWI within $48 \mathrm{~h}$ post-injury) of 26 patients, Schaefer and colleagues ${ }^{14}$ reported a weak correlation between lesions defined as any signal intensityabnormality in basal ganglia and/or thalamus and GCS score. Neither of these small studies, however, differentiated between uniand bilateral lesions, and they could not demonstrate any association between brainstem TAI and GCS scores.

On the other hand, a TBI study of pigs ${ }^{36}$ demonstrated association between TAI in the brainstem and lowered consciousness $8 \mathrm{~h}$ after trauma. No such association for the overall load of TAI was shown. Moreover, a study by Firsching and coworkers ${ }^{37}$ showed that duration of coma was longer in patients with any type of bilateral brainstem lesions than in patients with unilateral brainstem or supratentorial lesions.

The current study found that bilateral lesions in the thalamus were far more indicative of a low GCS score than any other MRI finding. This could imply that the thalamus is more central to arousal than the brainstem, and/or that bilateral damage here only occurs in conjunction with widespread lesions elsewhere in the brain. The study also showed, however, that patients with bilateral TAI lesions in the thalamus tended to have low GCS scores regardless of whether or not they had bilateral TAI lesions in the brainstem. The intralaminar nuclei of the thalamus receive multiple converging projections of the ARAS, which are diffusely projected to the cortex. ${ }^{34}$ Indeed, there are only two locations in the brain 


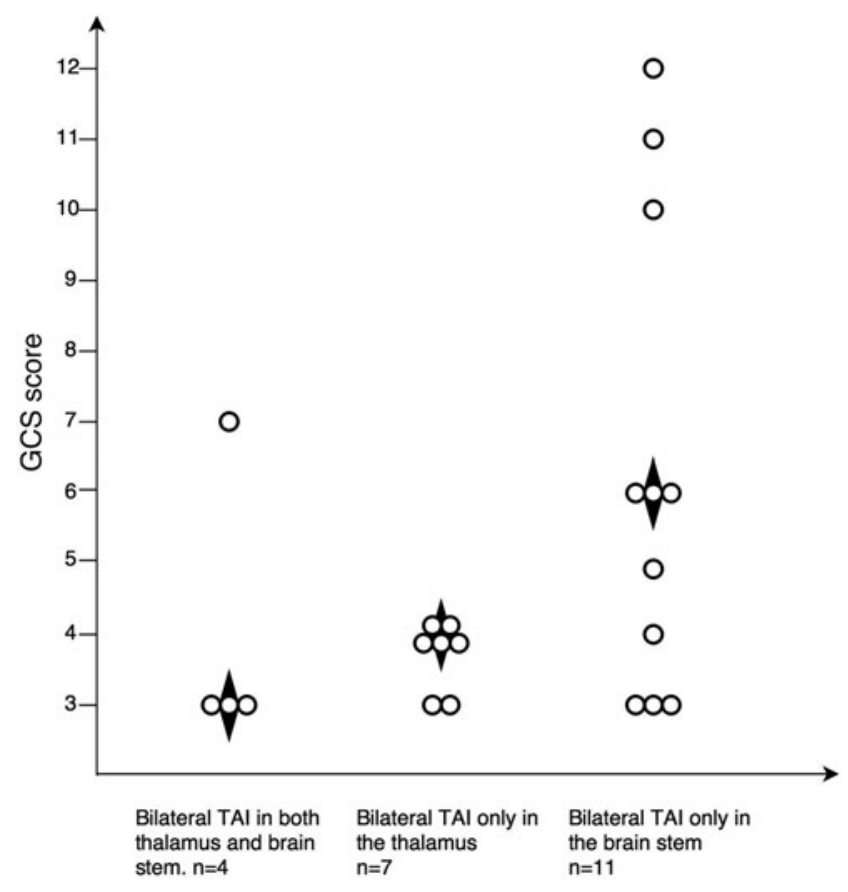

FIG. 4. Multiplicity of bilateral traumatic axonal injury (TAI) lesions in the thalamus and brainstem and relation to Glasgow Coma Scale (GCS) score. The figure separates out the four patients with bilateral lesions in both thalamus and brainstem. Median GCS score.

where very small bilateral lesions are known to affect consciousness: the mesencephalic reticular formation and the intralaminar thalamic nuclei. $^{38}$

Other parts of the thalamus may also be important for consciousness, ${ }^{39}$ because the thalamus has reciprocal connection to nearly every part of the brain. ${ }^{38}$ A clinical example is the loss of consciousness in bilateral paramedian thalamic stroke, ${ }^{40}$ and the recent findings that the thalamus is a critical and common site of action for anesthetic drugs. ${ }^{41}$ Also, in patients with TBI in the vegetative state/unresponsive wakefulness syndrome, both extensive TAI and thalamic injury are reported. ${ }^{42}$ Therefore, including TAI lesions in the subcortical nuclei in the definition and classification of TAI would be critical to better understand GCS scores.

This study also demonstrated that patients with TAI lesions in the basal ganglia had lower GCS scores than patients without such lesions. A recent study reported that clinical measures of awareness and wakefulness after trauma were associated with atrophy in both the thalamus and basal ganglia. Further, the arousal component of these measures was associated with atrophy in the bilateral putamen and globus pallidus. ${ }^{43}$ Because the current study had few patients with lesions in the basal ganglia, it is too small to address properly the question of how bilateral lesions in the basal ganglia affect consciousness, but the results suggest that lesions in the basal ganglia were associated with decreased GCS scores.

Interestingly, this study found that a large proportion (36-48\%) of patients with unilateral lesions in the thalamus, basal ganglia, and/or brainstem had GCS scores $>8$. Among patients with a GCS score of 13, almost one third had either unilateral TAI in the thalamus, basal ganglia, and/or brainstem. The neuroanatomic correlates of consciousness are distributed on both sides of the midline, and the brain has both intra- and interhemispheric connectivity. Hence, some redundancy of signaling pathways is provided. This might explain why the patients with only unilateral visible damage to these structures had higher GCS scores, and why patients with bilateral lesions in deep gray matter had such low levels of consciousness. It is also in line with our finding of worse outcome in patients with bilateral thalamic TAI compared with those with only unilateral thalamic lesions.

Lesions in the thalamus and, to even a greater degree, those in the brainstem were seldom detected on CT. In contrast, lesions in the basal ganglia were visible on $\mathrm{CT}$ in a somewhat higher percentage of patients. A previous CT study has also demonstrated findings of TAI in the basal ganglia and brainstem in a very low percentage of patients with TBI. ${ }^{44}$ We believe, however, that our comparison of uni- and bilateral MRI and CT findings in these subcortical nuclei in a considerable number of patients also is a novel finding.

\section{GCS score in relation to location of TAI lesions by MRI sequence}

For patients with thalamic lesions, both bilateral T2*GRE and FLAIR lesions were more indicative of a lower GCS score than the corresponding unilateral lesions. Patients with bilateral brainstem T2*GRE lesions had lower GCS scores compared with those with unilateral lesions, while this was less evident for FLAIR lesions. The results can also be explained, however, by lack of power because of small groups in the bilateral lesion category.

Hypointense foci depicted on T2*GRE-imaging represent magnetic susceptibility effects from breakdown products of hemoglobin and indicate micro-hemorrhagic TAI lesions. ${ }^{45}$ The FLAIR sequence and DWI sequence, however, show nonhemorrhagic TAI lesions, which may represent both vasogenic and cytotoxic edema in the acute stage of TBI. ${ }^{46}$ Hence, there may be different pathophysiological processes involved in the occurrence of micro-hemorrhages and edema. Although diffusion tensor imaging is assumed to better reflect the axonal injury, regions of interest within visible TAI lesions on early clinical MRI have also been found to demonstrate low fractional anisotropy values in the chronic phase compared with regions without such lesions. ${ }^{47}$

\section{Regression analyses of GCS scores}

The univariable ordinal regression analyses showed that patients with bilateral lesions in the thalamus, basal ganglia, and brainstem had the highest ORs for decreased GCS scores. Lower ORs were found in descending order in patients with TAI in the corpus callosum (TAI grade 2), unilateral lesions in the thalamus, basal ganglia, and brainstem, and finally in patients with TAI lesions in hemispheric white matter (TAI grade 1).

The BAC, in contrast to our previous study, ${ }^{24}$ had no effect on the GCS score. This might be because of some selection to this MRI study resulting in fewer patients with high BAC in the included group. Further, compressed or absent basal cisterns, which could reflect increased ICP also in some patients without mass lesions, were not associated with the GCS score. This lack of association probably can be explained by the fact that few patients had compressed cisterns and very few had absent basal cisterns in our cohort.

The standard TAI grading does not differ between unilateral and bilateral lesions in the brainstem and does not include TAI lesions in the thalamus or basal ganglia. ${ }^{4}$ Recent findings also suggests that TAI grade 3, the worst degree of TAI, does not necessarily imply a bad prognosis. ${ }^{48}$ A modified TAI grading with lesions in the substantia nigra and the mesencephalic tegmentum as a separate grade 
Table 4. Glasgow Coma Scale Scores and location of Uni-And Bilateral Traumatic Axonal Injury Lesions in Different Magnetic Resonance Imaging Sequences

\begin{tabular}{|c|c|c|c|c|c|c|c|c|}
\hline \multirow[b]{2}{*}{$\begin{array}{l}\text { Location and } \\
\text { MRI sequence }\end{array}$} & \multicolumn{2}{|c|}{$\begin{array}{c}\text { Patients without } \\
\text { TAI lesions }\end{array}$} & \multicolumn{2}{|c|}{$\begin{array}{c}\text { Patients with unilateral } \\
\text { TAI lesions }\end{array}$} & \multirow[b]{2}{*}{$\mathrm{p}$ value $^{\mathrm{a}}$} & \multicolumn{2}{|c|}{$\begin{array}{c}\text { Patients with bilateral } \\
\text { TAI lesions }\end{array}$} & \multirow[b]{2}{*}{$\mathrm{p}$ value $^{\mathrm{b}}$} \\
\hline & $\mathrm{n}$ & $\begin{array}{c}\text { Median } G C S \\
\text { score }(I Q R)\end{array}$ & $\mathrm{n}$ & $\begin{array}{c}\text { Median GCS } \\
\text { score }(I Q R)\end{array}$ & & $\mathrm{n}$ & $\begin{array}{c}\text { Median GCS } \\
\text { score }(I Q R)\end{array}$ & \\
\hline \multicolumn{9}{|l|}{ Thalamus } \\
\hline$-\mathrm{T} 2 * \mathrm{GRE}$ & 127 & $10(7-12)$ & 18 & $6.5(3.8-10)$ & 0.006 & 5 & $4(3-4)$ & 0.038 \\
\hline - FLAIR & 138 & $10(7-12)$ & 11 & $6(5-10)$ & 0.084 & 9 & $3(3-4)$ & 0.005 \\
\hline - DWI & 144 & $9.5(7-12)$ & 9 & $6(3.5-9.5)$ & 0.040 & 1 & $3(3-3)$ & 0.210 \\
\hline \multicolumn{9}{|l|}{ Basal ganglia } \\
\hline$-\mathrm{T} 2 * \mathrm{GRE}^{\mathrm{c}}$ & 126 & $10(7-12)$ & 22 & $7(5-11)$ & 0.044 & 3 & $4(3-4)$ & 0.084 \\
\hline - FLAIR & 148 & $10(7-12)$ & 10 & $5(3-6.5)$ & $<0.001$ & 0 & N.A. & N/A \\
\hline - DWI & 150 & $9(7-12)$ & 4 & $6(3.8-9.8)$ & 0.124 & 0 & N.A. & N/A \\
\hline \multicolumn{9}{|l|}{ Brainstem } \\
\hline$-\mathrm{T} 2 * \mathrm{GRE}$ & 119 & $10(7-13)$ & 21 & $9(5.5-10.5)$ & 0.064 & 10 & $4.5(3-6.3)$ & 0.008 \\
\hline - FLAIR & 128 & $10(7-12)$ & 20 & $7.5(4.3-10)$ & 0.025 & 10 & $5(3-8)$ & 0.162 \\
\hline - DWI & 137 & $10(7-12)$ & 14 & $6(4-8.3)$ & $<0.001$ & 3 & $3(3-3)$ & 0.126 \\
\hline
\end{tabular}

MRI, magnetic resonance imaging; TAI, traumatic axonal injury; GCS, Glasgow Coma Scale; IQR, interquartile range; T2*GRE, T2 weighted gradient echo imaging; FLAIR, fluid attenuated inversion recovery imaging; DWI, diffusion weighted imaging;

${ }^{a}$ Median GCS score in patients with unilateral TAI lesions compared with patients without TAI lesions.

${ }^{b}$ Median GCS score in patients with bilateral TAI lesions compared with patients with unilateral TAI lesions.

${ }^{\mathrm{c}}$ In one patient, T2*GRE image quality was too poor to conclude on TAI any other place than in the basal ganglia.

has recently been proposed. ${ }^{49}$ The current study, however, provides novel information suggesting that the TAI grading can be refined and improved by including lesions in deep gray matter nuclei, as well as the presence of uni- and bilateral TAI lesions in deeper parts of the brain. This would provide an opportunity to better understand the severity of TBI in the acute phase.

\section{Strengths and limitations}

Both the prospective data collection with an overview of the entire consecutive cohort and the size of the MRI cohort are strengths of this study. Further, the image reading was conducted blinded for clinical information and the interrater agreement was found to be good to excellent in an earlier study. ${ }^{21}$ The exclusion of patients with intracranial mass lesions removed a possible confounder for lowered consciousness.

There are limitations to this study. First, although more than three fourths had MRI within two weeks, the time between injury and MRI varied across the patients. This may influence the results of DWI analyses and, to some degree, the FLAIR analyses. On the other hand, T2*GRE lesions have been shown to persists after three months. ${ }^{21}$ It is also worth noting that performing very early MRI on

Table 5. Univariable Analyses: Glasgow Coma Scale score* and Association to Magnetic Resonance Imaging and Computed Tomography Findings and Some Other Clinical Variables

\begin{tabular}{|c|c|c|c|}
\hline Variable & Location & OR $(95 \% C I)$ & $\mathrm{p}$ value \\
\hline \multirow[t]{11}{*}{ MRI findings (any sequence) } & Thalamus & & \\
\hline & - Bilateral & $35.8(10.5-121.8)$ & $<0.001$ \\
\hline & - Unilateral & $3.9(1.6-9.5)$ & 0.003 \\
\hline & Basal ganglia & & \\
\hline & - Bilateral & $13.1(2.0-88.2)$ & 0.008 \\
\hline & - Unilateral & $2.8(1.3-6.1)$ & 0.008 \\
\hline & Brainstem (TAI grade 3$)^{\mathrm{a}}$ & & \\
\hline & - Bilateral & $11.4(4.0-32.2)$ & $<0.001$ \\
\hline & - Unilateral & $2.7(1.3-5.4)$ & 0.005 \\
\hline & Corpus callosum (TAI grade 2$)^{\mathrm{a}}$ & $4.9(2.1-11.5)$ & $<0.001$ \\
\hline & Hemispheric white matter (TAI grade 1$)^{\mathrm{a}}$ & $1.4(0.6-3.2)$ & 0.446 \\
\hline \multirow[t]{2}{*}{ CT findings } & Compressed or absent basal cisterns ${ }^{\mathrm{b}}$ & $1.7(0.8-3.6)$ & 0.190 \\
\hline & Rotterdam CT score & $1.8(1.3-2.6)$ & 0.001 \\
\hline \multirow[t]{2}{*}{ Other } & $\mathrm{BAC}(\mathrm{mmol} / \mathrm{L})^{\mathrm{c}}$ & $1.01(0.99-1.02)$ & 0.288 \\
\hline & Injury Severity Score & $1.11(1.08-1.14)$ & $<0.001$ \\
\hline
\end{tabular}

OR, odds ratio; CI, confidence interval; MRI, magnetic resonance imaging; TAI, traumatic axonal injury; CT, computed tomography; BAC, blood alcohol concentration.

* Glasgow Coma Scale score is inverted for these analyses.

${ }^{a}$ Irrespective of TAI lesions in thalamus or basal ganglia.

${ }^{\mathrm{b}} 19$ patients had compressed basal cisterns, four patients had absent basal cisterns.

${ }^{\mathrm{c}} 126$ of the patients were tested for blood alcohol content at admission. 
these patients is challenging, and including only patients with MRI in the very acute phase would increase the selection bias substantially. Despite the somewhat later MRI, the current results still demonstrated that these clinical MRI biomarkers of TAI during the first weeks were associated with GCS scores before intubation or at admission. We also found that patients with high GCS scores were examined with MRI earlier than those with low GCS scores. Hence, it is less likely that bilateral lesions were missed in this group consisting of more moderate patients with TBI.

Second, an even larger study population would have been preferable, because the number of patients with bilateral TAI in subcortical gray matter was quite small. Third, further analyses of combining lesion load in the different sequences could have more clearly pointed out whether bilaterality in itself could explain lower GCS scores. Fourth, 1.5 Tesla MRI scanners have reduced sensitivity for lesion detection compared with 3 Tesla scanners. ${ }^{50}$ The 1.5 Tesla, however, is the most commonly used in the clinic. Also, susceptibility weighted imaging (SWI) has been found to be more sensitive to detect micro-hemorrhagic lesions after TBI than T2* GRE. ${ }^{49}$ In the study of Geurts and colleagues ${ }^{16}$ using $3 \mathrm{~T}$ at median seven weeks, they counted more than twice the number of punctate lesions (assumed to be TAI lesions) in subcortical gray matter or brainstem by using SWI compared with T2*GRE. This might imply that a revised cutoff value (e.g., $>1-2$ lesions per location) needs to be considered if using 3T SWI as a TAI biomarker in a new grading. Further, SWI sequences have limitations; for instance, the number of SWI lesions detected may vary between scanner vendors as the "blooming" effect can differ.

\section{Conclusion}

This Trondheim TBI study demonstrated that in patients with TBI without mass lesions, bilateral TAI lesions in the thalamus were associated with the lowest level of consciousness in the very acute phase. Next came patients with bilateral TAI lesions in the basal ganglia and/or brainstem. Further, patients without any lesions in these locations had higher GCS scores than those with unilateral lesions. Unilateral TAI lesions on MRI, however, were still found in almost one third of patients with a GCS score of 13. In addition, the study demonstrated that TAI biomarkers in the thalamus and brainstem very seldom were detected on CT.

A future TAI-MRI grading also including such subcortical bilateral lesions as a separate and worst grade could provide clinicians with important information in the acute phase of moderate and severe TBI. A possible new TAI grading should be evaluated further in larger study samples by advanced statistical models for its value in prediction of outcome, followed by validation in largescale multicenter studies like the ongoing CENTER-TBI study.

\section{Acknowledgments}

We thank Mari Folvik, Jana Rydland, and Jian Xu for their contributions in the MRI readings. We would also like to thank Anne-Mari Holte Flusund for her work in examining CT scans for lesions in the thalamus, basal ganglia, and brainstem. HKM received a research scholarship from the Norwegian Research Council and funding from the Norwegian University of Science and Technology (NTNU). KGM and TS have, during the study period, received research grants from the liaison committee between the Central Norway regional health authority and NTNU. This work (AMF) has also been partly funded by ERA-NET NEURON and the Research Council of Norway (TAI-MRI project).

\section{Author Disclosure Statement}

No competing financial interests exist.

\section{References}

1. Skandsen, T., Kvistad, K.A., Solheim, O., Strand, I.H., Folvik, M., and Vik, A. (2010). Prevalence and impact of diffuse axonal injury in patients with moderate and severe head injury: a cohort study of early magnetic resonance imaging findings and 1-year outcome. J. Neurosurg. 113, 556-563.

2. Adams, J.H., Doyle, D., Ford, I., Gennarelli, T.A., Graham, D.I., and Mclellan, D.R. (1989). Diffuse axonal injury in head injury: Definition, diagnosis and grading. Histopathology 15, 49-59.

3. Murray, J.G., Gean, A.D., and Evans, S.J. (1996). Imaging of acute head injury. Semin. Ultrasound CT MR 17, 185-205.

4. Gentry, L.R. (1994). Imaging of closed head injury. Radiology 191, 1-17.

5. Ommaya, A.K., and Gennarelli, T.A. (1974). Cerebral concussion and traumatic unconsciousness. Correlation of experimental and clinical observations on blunt head injuries. Brain 97, 633-654.

6. Bramlett, H.M., Kraydieh, S., Green, E.J., and Dietrich, W.D. (1997). Temporal and regional patterns of axonal damage following traumatic brain injury: a beta-amyloid precursor protein immunocytochemical study in rats. J. Neuropathol. Exp. Neurol. 56, 1132-1141.

7. Lifshitz, J., Kelley, B.J., and Povlishock, J.T. (2007). Perisomatic thalamic axotomy after diffuse traumatic brain injury is associated with atrophy rather than cell death. J. Neuropathol. Exp. Neurol. 66, 218-229.

8. Moen, K.G., Brezova, V., Skandsen, T., Håberg, A.K., Folvik, M., and Vik, A. (2014). Traumatic axonal injury: the prognostic value of lesion load in corpus callosum, brain stem, and thalamus in different magnetic resonance imaging sequences. J. Neurotrauma 31, 14861496.

9. Teasdale, G., Maas, A., Lecky, F., Manley, G., Stocchetti, N., and Murray, G. (2014). The Glasgow Coma Scale at 40 years: standing the test of time. Lancet Neurol. 13, 844-854.

10. Moruzzi, G., and Magoun, H.W. (1949). Brainstem reticular formation and activation of the EEG. Elecroencephalogr. Clin. Neurophysiol. 1, 455-473.

11. Laureys, S., and Schiff, N.D. (2012). Coma and consciousness: paradigms (re)framed by neuroimaging. Neuroimage 61, 478-491.

12. Majerus, S., Gill-Thwaites, H., Andrews, K., and Laureys, S. (2005). Behavioral evaluation of consciousness in severe brain damage. Prog. Brain Res. 150, 397-413.

13. Skandsen, T., Kvistad, K.A., Solheim, O., Lydersen, S., Strand, I.H., and Vik, A. (2011). Prognostic value of magnetic resonance imaging in moderate and severe head injury: a prospective study of early MRI findings and one-year outcome. J. Neurotrauma 28, 691-699.

14. Schaefer, P.W., Huisman, T.A., Sorensen, A.G., Gonzalez, R.G., and Schwamm, L.H. (2004). Diffusion-weighted MR imaging in closed head injury: high correlation with initial glasgow coma scale score and score on modified Rankin scale at discharge. Radiology 233, 58-66.

15. Gerber, D.J., Weintraub, A.H., Cusick, C.P., Ricci, P.E., and Whiteneck, G.G. (2004). Magnetic resonance imaging of traumatic brain injury: relationship of $\mathrm{T} 2 * \mathrm{SE}$ and $\mathrm{T} 2 \mathrm{GE}$ to clinical severity and outcome. Brain Inj. 18, 1083-1097.

16. Geurts, B.H., Andriessen, T.M., Goraj, B.M., and Vos, P.E. (2012). The reliability of magnetic resonance imaging in traumatic brain injury lesion detection. Brain Inj. 26, 1439-1450.

17. Grados, M.A., Slomine, B.S., Gerring, J.P., Vasa, R., Bryan, N., and Denckla, M.B. (2001). Depth of lesion model in children and adolescents with moderate to severe traumatic brain injury: use of SPGR MRI to predict severity and outcome. J. Neurol. Neurosurg. Psychiatry 70, 350-358.

18. Levin, H.S., Williams, D., Crofford, M.J., High, W.M. Jr., Eisenberg, H.M., Amparo, E.G., Guinto, F.C. Jr., Kalisky, Z., Handel, S.F., and Goldman, A.M. (1988). Relationship of depth of brain lesions to consciousness and outcome after closed head injury. J. Neurosurg. 69, 861-866.

19. Anderson, C.V., Wood, D.M., Bigler, E.D., and Blatter, D.D. (1996). Lesion volume, injury severity, and thalamic integrity following head injury. J. Neurotrauma 13, 59-65.

20. Schonberger, M., Ponsford, J., Reutens, D., Beare, R., and O'Sullivan, R. (2009). The relationship between age, injury severity, and MRI findings after traumatic brain injury. J. Neurotrauma 26, 2157-2167. 
21. Moen, K.G., Skandsen, T., Folvik, M., Brezova, V., Kvistad, K.A., Rydland, J., Manley, G.T., and Vik, A. (2012). A longitudinal MRI study of traumatic axonal injury in patients with moderate and severe traumatic brain injury. J. Neurol. Neurosurg. Psychiatry 83, 1193-200.

22. Stein, S.C., and Spettell, C. (1995). The Head Injury Severity Scale (HISS): a practical classification of closed-head injury. Brain Inj. 9, 437-444.

23. Matsusue, E., Sugihara, S., Fujii, S., Ohama, E., Kinoshita, T., and Ogawa, T. (2006). White matter changes in elderly people: MRpathologic correlations. Magn. Reson. Med. Sci. 5, 99-104.

24. Rundhaug, N.P., Moen, K.G., Skandsen, T., Schirmer-Mikalsen, K. Lund, S.B., Hara, S., and Vik, A. (2015). Moderate and severe traumatic brain injury: effect of blood alcohol concentration on Glasgow Coma Scale score and relation to computed tomography findings. J. Neurosurg. 122, 211-218.

25. Lange, R.T., Iverson, G.L., Brubacher, J.R., and Franzen, M.D. (2010). Effect of blood alcohol level on Glasgow Coma Scale scores following traumatic brain injury. Brain Inj. 24, 919-927.

26. Marshall, L.F., Marshall, S.B., Klauber, M.R., van Berkum Clark, M., Eisenberg, H.M., Jane, J.A., Luerssen, T.G., Marmarou, A., and Foulkes, M.A. (1991). A new classification of head injury based on computerized tomography. J. Neurosurg. 75, S14-S20.

27. Maas, A.I., Hukkelhoven, C.W., Marshall, L.F., and Steyerberg, E.W (2005). Prediction of outcome in traumatic brain injury with computed tomographic characteristics: A comparison between the computed tomographic classification and combinations of computed tomographic predictors. Neurosurgery $57,1173-1182$.

28. Jennett, B., Snoek, J., Bond, M.R., and Brooks, N. (1981). Disability after severe head injury: observations on the use of the Glasgow Outcome Scale. J. Neurol. Neurosurg. Psychiatry 44, 285-293.

29. de Leeuw, F.E., de Groot, J.C., Achten, E., Oudkerk, M., Ramos, L.M., Heijboer, R., Hofman, A., Jolles, J., van Gijn, J., and Breteler, M.M. (2001). Prevalence of cerebral white matter lesions in elderly people: a population based magnetic resonance imaging study. The Rotterdam Scan Study. J. Neurol. Neurosurg. Psychiatry 70, 9-14.

30. Fazekas, F., Chawluk, J.B., Alavi, A., Hurtig, H., and Zimmerman, R.A. (1987). MR signal abnormalities at $1.5 \mathrm{~T}$ in Alzheimer's dementia and normal aging. Am. J. Neuroradiol. 8, 421-426.

31. Bernat, J.L. (2006). Chronic disorders of consciousness. Lancet 367 1181-1192.

32. Zeman, A. (2001). Consciousness. Brain 124, 1263-1289.

33. Schiff, N.D. (2016). Central thalamic deep brain stimulation to support anterior forebrain mesocircuit function in the severely injured brain. J. Neural Transm. 123, 797-806.

34. Posner, J.B., and Plum, F. (2007). Plum and Posner's Diagnosis of Stupor and Coma. Oxford University Press: New York.

35. Parvizi, J., and Damasio, A. (2001). Consciousness and the brainstem. Cognition 79, 135-160.

36. Smith, D.H., Nonaka, M., Miller, R., Leoni, M., Chen, X.H., Alsop, D., and Meaney, D.F. (2000). Immediate coma following inertial brain injury dependent on axonal damage in the brainstem. J. Neurosurg. 93, 315-322.

37. Firsching, R., Woischneck, D., Klein, S., Reißberg, S., Döhring, W., and Peters, B. (2001). Classification of severe head injury based on magnetic resonance imaging. Acta Neurochir. (Wien) 143, 263-271.

38. Ward, L.M. (2011). The thalamic dynamic core theory of conscious experience. Conscious. Cogn. 20, 464-486.
39. Laureys, S., Faymonville, M.E., Luxen, A., Lamy, M., Franck, G., and Maquet, P. (2000). Restoration of thalamocortical connectivity after recovery from persistent vegetative state. Lancet 355, 1790-1791.

40. Zappella, N., Merceron, S., Nifle, C., Hilly-Ginoux, J., Bruneel, F Troche, G., Cordoliani, Y.S., Bedos, J.P., Pico, F., and Legriel, S. (2014). Artery of Percheron infarction as an unusual cause of coma: three cases and literature review. Neurocrit. Care 20, 494-501.

41. Alkire, M.T., and Miller, J. (2005). General anesthesia and the neural correlates of consciousness. Prog. Brain Res. 150, 229-244.

42. Jennett, B., Adams, J.H., Murray, L.S., and Graham, D.I. (2001). Neuropathology in vegetative and severely disabled patients after head injury. Neurology 56, 486-490.

43. Lutkenhoff, E.S., Chiang, J., Tshibanda, L., Kamau, E., Kirsch, M., Pickard, J.D., Laureys, S., Owen, A.M., and Monti, M.M. (2015). Thalamic and extrathalamic mechanisms of consciousness after severe brain injury. Ann. Neurol. 78, 68-76.

44. Nelson, D.W., Nyström, H., MacCallum, R.M., Thornquist, B., Lilja, A., Bellander, B.M., Rudehill, A., Wanecek, M., and Weitzberg, E. (2010). Extended analysis of early computed tomography scans of traumatic brain injured patients and relations to outcome. J. Neurotrauma 27, 51-64.

45. Messori, A., Polonara, G., Mabiglia, C., and Salvolini, U. (2003). Is haemosiderin visible indefinitely on gradient-echo MRI following traumatic intracerebral haemorrhage? Neuroradiology 45, 881-886.

46. Parizel, P.M., Van Goethem, J.W., Özsarlak, O. Maes, M., and Phil lips, C.D. (2005). New developments in the neuroradiological diagnosis of craniocerebral trauma. Eur. Radiol. 15, 569-581.

47. Moen, K.G., Vik, A., Olsen, A., Skandsen, T., Håberg, A.K., Evensen, K.A., and Eikenes, L. (2016). Traumatic axonal injury: Relationships between lesions in the early phase and diffusion tensor imaging parameters in the chronic phase of traumatic brain injury. J. Neurosci. Res. 94, 623-635.

48. Izzy, S., Mazwi, N.L., Martinez, S., Spencer, C.A., Klein, J.P., Parikh, G., Glenn, M.B., Greenberg, S.M., Greer, D.M., Wu, O., and Edlow, B.L. (2017). Revisiting grade 3 diffuse axonal injury: not all brainstem microbleeds are prognostically equal. Neurocrit. Care 27, 199-207.

49. Abu Hamdeh, S., Marklund, N., Lannsjo, M., Howells, T., Raininko, R., Wikstrom, J., and Enblad, P. (2017). Extended anatomical grading in diffuse axonal injury using MRI: hemorrhagic lesions in the substantia nigra and mesencephalic tegmentum indicate poor long-term outcome. J. Neurotrauma 34, 341-352.

50. Scheid, R., Ott, D.V, Roth, H., Schroeter, M.L., and von Cramon, D.Y. (2007). Comparative magnetic resonance imaging at 1.5 and 3 Tesla for the evaluation of traumatic microbleeds. J. Neurotrauma 24, 18111816.

Address correspondence to:

Hans Kristian Moe

Department of Neuromedicine and Movement Science Norwegian University of Science and Technology

Edvard Griegs Gate 8

7030 Trondheim

Norway

E-mail: hanskristianmoe91@gmail.com 